Revista Perspectivas Online: Biológicas \& Saúde Novembro/2020, v.10, n.35, p.63-74 ISSN: 2236-8868 (Online) DOI: $10.25242 / / 8868103520202035$

\title{
ASPECTOS NEUROPSICOLÓGICOS DE UNIVERSITÁRIOS COM ANSIEDADE SOCIAL
}

\section{Lucas Emmanuel Lopes e Santos ${ }^{1 *}$, Marina de Souza Guzzo ${ }^{2}$, Carlos Alexandre Antunes Cardoso $^{2}$, Fernanda Lemes Batista Magalhães ${ }^{2} \&$ Cecília Souza Oliveira $^{2}$}

SANTOS, L.E.L.; GUZZO, M.S.; CARDOSO, C.A.A.; MAGALHÃES, F.L.B.; \& OLIVEIRA, C.S. Aspectos neuropsicológicos de universitários com ansiedade social. Perspectivas Online: Biológicas \& Saúde, v.10, n.35, p.63-74, 2020.

\section{RESUMO}

O Transtorno de Ansiedade Social (TAS) é definido por um padrão acentuado de alarme, medo e desconforto desencadeado pela exposição a situações nas quais o indivíduo deve interagir, realizar tarefas sob a avaliação de outras pessoas ou engajar-se em atividades sociais. $\mathrm{O}$ presente estudo teve como objetivo caracterizar o perfil clínico e neuropsicológico de universitários com sintomas de ansiedade social. Trata-se de uma pesquisa do tipo caso-controle realizada em três etapas: 1) utilizou-se a Escala de Ansiedade Social de Liebowitz (LSAS) como instrumento de rastreio da prevalência dos sintomas na amostra estudada; 2) os participantes responderam a Entrevista Clínica Estruturada para os Transtornos do DSM-5 (SCID-5), a fim de coletar dados que corroborassem os critérios diagnósticos para o TAS; 3) os grupos caso e controle foram avaliados em termos de desempenho cognitivo por meio de uma bateria de testes neuropsicológicos computadorizados. Observou-se que o grupo caso obteve um escore total médio de 72,91 na LSAS, ao passo que o grupo controle obteve uma média de 49,42 pontos na escala. No que se refere ao desempenho cognitivo, foi possível corroborar os achados da literatura científica que apontam para prejuízos decorrentes do Transtorno de Ansiedade Social em funções neuropsicológicas específicas, como a atenção seletiva, habilidades visuoespaciais e de resolução de problemas complexos.

Palavras-chave: Fobia social; Avaliação neuropsicológica; Perfil cognitivo.

\footnotetext{
${ }^{1}$ Faculdade de Medicina de Ribeirão Preto da Universidade de São Paulo - FMRP/USP . Av. Bandeirantes, 3900 - Vila Monte Alegre, Ribeirão Preto - SP, CEP: 14049-900, Brasil.

${ }^{2}$ Universidade Federal Fluminense - UFF Campos - Rua José do Patrocínio, n 71 , Centro, Campos dos Goytacazes - RJ, CEP 28010-385, Brasil.

$\left(^{*}\right)$ e-mail: lucaselopes16@gmail.com
} 
Online Perspectives Journal: Biological \& Health November/2020, v.10, n.35, p.63-74 ISSN: 2236-8868 (Online) DOI: $10.25242 / / 8868103520202035$

\title{
NEUROPSYCHOLOGICAL ASPECTS OF COLLEGE STUDENTS WITH SOCIAL ANXIETY
}

\section{Lucas Emmanuel Lopes e Santos ${ }^{1 *}$, Marina de Souza Guzzo $^{2}$, Carlos Alexandre Antunes Cardoso $^{2}$, Fernanda Lemes Batista Magalhães ${ }^{2} \&$ Cecília Souza Oliveira $^{2}$}

SANTOS, L.E.L.; GUZZO, M.S.; CARDOSO, C.A.A.; MAGALHÃES, F.L.B.; \& OLIVEIRA, C.S. Neuropsychological aspects of college students with social anxiety. Online Perspectives: Biological \& Health, v.10, n.35, p.63-74, 2020.

\begin{abstract}
Social Anxiety Disorder (SAD) is defined by an intense pattern of alarm, fear, and discomfort triggered by exposure to situations in which the individual must interact, perform tasks under the evaluation of others, or engage in social activities. The present study aimed to characterize the clinical and neuropsychological profile of college students with symptoms of social anxiety. This is a case-control study conducted in three stages: 1) the Liebowitz Social Anxiety Scale (LSAS) was used as a screening tool for the prevalence of symptoms in the sample; 2) the Structured Clinical Interview for DSM-5 (SCID-5) was administered to collect data in order to corroborate the diagnostic criteria for

$\mathrm{SAD} ; 3)$ the case and control groups were assessed in terms of cognitive performance by a battery of computerized neuropsychological tests. We observed that the case group had an average total score of 72.91 on the LSAS, whereas the control group had an average of 49.42 points on the scale. With regard to cognitive performance, there was a statistically significant difference in the mean response time between the case and control groups. It was possible to corroborate the findings of the scientific literature that point to impairments caused by SAD in specific neuropsychological functions, such as selective attention, visuospatial abilities and complex problem-solving.
\end{abstract}

Keywords: Social phobia; Neuropsychological assessment; Cognitive profile.

\footnotetext{
${ }^{1}$ Faculdade de Medicina de Ribeirão Preto da Universidade de São Paulo - FMRP/USP . Av. Bandeirantes, 3900 - Vila Monte Alegre, Ribeirão Preto - SP, CEP: 14049-900, Brasil.

${ }^{2}$ Universidade Federal Fluminense - UFF Campos - Rua José do Patrocínio, n 71, Centro, Campos dos Goytacazes - RJ, CEP 28010-385, Brasil.

$(*)$ e-mail: lucaselopes16@gmail.com
} 


\section{INTRODUÇÃO}

Os transtornos de ansiedade podem ser definidos como um padrão intenso e crônico de medo com relação a eventos futuros, que prejudica sobremaneira o desempenho de indivíduos em situações cotidianas como o trabalho, a escola e as interações sociais. Em termos evolutivos, o medo é a resposta emocional do organismo frente a uma ameaça iminente. No entanto, a experiência pode se tornar patológica quando a sensação de medo for excessiva e/ou irreal, causando prejuízos funcionais para o indivíduo. Os sintomas fisiológicos mais comuns relacionados à ansiedade são taquicardia, sudorese, inquietação, tremores, insônia e dificuldade de concentração (CASTILLO ET AL, 2000).

Whitbourne e Halgin (2015) apontam que, dentre os transtornos psicológicos, os de ansiedade ocupam o segundo lugar de maior prevalência na população $(28,8 \%)$, atrás apenas dos transtornos por uso de substâncias. Já está bem estabelecido na literatura científica que a causa dos transtornos de ansiedade é multifatorial (EATON et al., 1989; LIMA, 1999; KESSLER et al., 1994). Os diversos ambientes familiares, classes sociais, estratos econômicos, culturais e sociais podem interferir como fatores de risco ou protetores para o desenvolvimento de um transtorno mental (CARLSON, 2002; CASTILLO et al., 2000; KANDEL et al., 2014; PIES, 1994; WHITE, 2005). Em um estudo de revisão, Asbahr (2004) relatou que pesquisas com famílias de gêmeos têm correlacionado o aumento da neurotransmissão serotoninérgica à etiologia dos transtornos de ansiedade, o que sugere uma possível influência de aspectos genéticos associados ao desenvolvimento do quadro clínico.

Apesar da expressão patológica do medo ser um aspecto comum dos transtornos de ansiedade (TA), diferenças com relação aos estímulos que evocam a ansiedade e as diversas respostas comportamentais dos indivíduos caracterizam os subtipos de TA: transtorno do pânico, agorafobia, transtorno obsessivo-compulsivo, transtorno de ansiedade generalizada, fobias específicas, transtorno de estresse pós-traumático e transtorno de ansiedade social (BEAR, CONNORS \& PARADISO, 2008).

O Transtorno de Ansiedade Social (TAS) é caracterizado por um padrão acentuado de alarme, medo e desconforto desencadeado pela exposição a situações nas quais o indivíduo deve interagir, realizar tarefas sob a avaliação de outras pessoas ou engajar-se em atividades sociais. Dentre os principais medos vivenciados por indivíduos com TAS estão o de parecerem ridículos, dizerem tolices, serem observados por outras pessoas, interagirem com estranhos ou pessoas do sexo oposto e serem o centro das atenções. Pacientes diagnosticados com TAS apresentam sintomas somáticos como palpitação, rubor, tremor e sudorese quando expostos a situações que exigem algum nível de interação social (BURATO, 2009).

Desde sua inclusão no Manual Diagnóstico e Estatístico de Transtornos Mentais (DSM-III), na década de 1980, o TAS tem despertado o interesse de pesquisadores e profissionais clínicos por também ser considerado um problema de saúde pública (ABOUSALEH et al., 2001, apud BAPTISTA et al., 2012), o qual demanda a criação de políticas nacionais para o enfrentamento deste e demais transtornos mentais (TALÃO et al., 2020). Segundo dados do National Comorbidity Survey Replication Study (NCS-R), 12,1\% dos norte-americanos são acometidos pelo TAS em algum momento da vida, sendo considerado o quarto transtorno psiquiátrico mais comum nos EUA (KESSLER et al., 2005, apud BARLOW, 2016).

Os primeiros sintomas do Transtorno de Ansiedade Social podem se manifestar ainda na infância ou adolescência e prevalecem durante a fase adulta (Schneier et al., 1992; Bruce et 
al., 2005). Entretanto, Scheiner et al. (1992) apontam para o fato de que a maioria dos indivíduos com TAS não busca tratamento especializado, a não ser nos casos em que há manifestação de comorbidades psiquiátricas. É válido ressaltar que cerca de $80 \%$ dos indivíduos com TAS cumprem os critérios diagnósticos para outros transtornos como fobia específica, agorafobia, depressão e transtornos relacionados ao uso de álcool, sendo que na maioria dos casos o TAS ocorre previamente ao problema comórbido (MAGEE et al., 1996; SCHNEIER et al., 1992).

Em um estudo realizado com universitários brasileiros com queixas de ansiedade social, Baptista (2006) verificou que a prevalência do TAS foi de $12,5 \%$ em mulheres e de $7,4 \%$ em homens. Além disso, constatou-se que a idade média de início do transtorno foi de 11,4 anos. Com relação à gravidade dos sintomas, $63,7 \%$ dos indivíduos foram classificados como apresentando TAS moderado, seguido por grau leve $(21,9 \%)$ e grave $(14,3 \%)$. A pesquisa também apontou que indivíduos com TAS faziam mais uso de medicamentos psicotrópicos $(5,5 \%)$ do que os sujeitos sem o transtorno $(2,2 \%)$, embora a maioria daqueles acometidos pela doença não estivesse em tratamento específico para o TAS.

Alguns dos prejuízos causados pelo Transtorno de Ansiedade Social já estão bem consolidados na literatura (ACARTURK, G.; VAN STRATEN, H.; CUIJIPERS, 2008, apud BARLOW, 2016). É consenso entre os indivíduos acometidos pelo transtorno que o desempenho em atividades cotidianas foi seriamente prejudicado devido à sua condição clínica. Evidencia-se que as implicações do TAS podem ocorrer em situações específicas da vida, como na área profissional, acadêmica e social. Entretanto, Sutterby (2009) aponta para a escassez de estudos empíricos que tenham investigado os mecanismos neurocognitivos do TAS, os quais poderiam contribuir para a elucidação de componentes preventivos e terapêuticos do transtorno.

Nas últimas décadas, a investigação na área da neuropsicologia tem fornecido parâmetros para a avaliação do funcionamento cognitivo em diversos quadros psiquiátricos. No que se refere aos transtornos de ansiedade, mais especificamente o TAS, Asmundson, Stein, Larsen e Walker (1994) foram pioneiros no estudo dos aspectos neuropsicológicos em uma amostra de pacientes com ansiedade social. Mais recentemente, Graver e White (2007) demonstraram prejuízos em tarefas de resolução de problemas e de funções atencionais em indivíduos diagnosticados com TAS. Conforme sugerido por Sutterby (2009), os primeiros achados da literatura acerca do desempenho neuropsicológico de indivíduos com Transtorno de Ansiedade Social foram escassos e inconsistentes, evidenciando a necessidade de pesquisas que sejam capazes de elucidar o perfil cognitivo associado a este transtorno. Nessa perspectiva, o presente estudo teve como objetivo caracterizar o perfil clínico e neuropsicológico de estudantes universitários com sintomas de ansiedade social.

\section{METODOLOGIA}

Trata-se de um estudo do tipo caso-controle realizado em três etapas: I) utilizou-se a Escala de Ansiedade Social de Liebowitz (LSAS) como instrumento de rastreio da prevalência dos sintomas em um grupo de universitários; II) os participantes responderam a Entrevista Clínica Estruturada para os Transtornos do DSM-5 (SCID-5), a fim de coletar dados que corroborassem os critérios diagnósticos para o TAS, descritos no Manual Diagnóstico e Estatístico de Transtornos Mentais (DSM-5); III) os grupos caso (i.e., universitários com TAS) e controle foram avaliados em termos de desempenho cognitivo por 
meio de uma bateria de testes neuropsicológicos computadorizados.

\subsection{Amostra e Aspectos éticos}

A amostra foi composta por estudantes de ambos os sexos da Universidade Federal Fluminense (UFF) - Campos dos Goytacazes. Foram incluídos na pesquisa universitários que demonstrassem interesse espontâneo de participação e que estivessem regularmente matriculados nos seguintes cursos de graduação da UFF: Psicologia, Economia, Serviço Social, História, Geografia e Ciências Sociais. Foram excluídos do estudo indivíduos que apresentassem algum déficit visual, auditivo ou motor que impedisse a aplicação da bateria computadorizada de testes neuropsicológicos. Após a avaliação clínica, 11 estudantes foram categorizados no grupo caso (média de idade 21,90 $\pm 4,08$ ) e 19 no grupo controle (média de idade $21,95 \pm 6,80$ ). A pesquisa foi aprovada pelo Comitê de Ética em Pesquisa com Seres Humanos ( $n^{\circ}$ 89847318.2.0000.5243) e o Termo de Consentimento Livre e Esclarecido foi assinado por todos os participantes.

\subsection{Instrumentos}

A Escala de Ansiedade Social de Liebowitz (LSAS) é um instrumento psicológico que avalia o grau de interferência da ansiedade em 24 situações cotidianas. É composta por duas subescalas: interação social (11 itens) e ansiedade de desempenho (13 itens), avaliados por meio da escala Likert (zero a três pontos). Diversas pesquisas têm comprovado a eficácia da LSAS, atribuindo boas qualidades psicométricas para o instrumento. Em um estudo realizado por Santos et al. (2015), os autores destacaram a boa capacidade discriminatória da LSAS para a avaliação de quadros clínicos e subclínicos em uma amostra de universitários.

A Entrevista Clínica Estruturada para os Transtornos do DSM (SCID-5) é composta por módulos que auxiliam o profissional na investigação das principais categorias diagnósticas descritas no Manual Diagnóstico e Estatístico de Transtornos Mentais, atualmente em sua quinta edição (DSM-5). Um estudo de confiabilidade desenvolvido por Del-Ben et al. (2001) demonstrou resultados psicométricos favoráveis para a versão traduzida e adaptada do instrumento para o português.

Para o exame das funções cognitivas, utilizou-se a bateria de testes PEBL Version 2.0 - The Psychology Experiment Building Language (MUELLER, 2014), que compreende um software livre, com código fonte gratuito, desenvolvido para a avaliação de diversos construtos psicológicos. É válido ressaltar que o Conselho Federal de Psicologia (CFP), por meio da Resolução $n^{\circ}$ 002/2003, permite a utilização de testes psicológicos ainda não padronizados para a população brasileira para fins de pesquisa científica. Para a avaliação das funções executivas e da memória de trabalho utilizou-se o Berg's Card Sorting Test (BCST), versão adaptada para o computador do Teste Wisconsin de Classificação de Cartas (HEATON et al., 1993). A avaliação da impulsividade, tempo de reação e atenção seletiva foi realizada através do Go/No-go Task e Oddball Task. O Traveling Salesman Problem (TSP) foi utilizado para a avaliação do processamento visual e planejamento espacial. Por fim, avaliou-se o desempenho dos indivíduos em tarefas de memória visuoespacial de curto prazo através do Corsi Blocks. 


\subsection{Procedimentos}

Todas as três etapas da pesquisa foram realizadas no período entre agosto e setembro de 2018. Cada encontro com os universitários em sala de aula durou cerca de 30 minutos, ocasião em que os participantes preencheram a LSAS. Após a triagem pela Escala de Ansiedade Social de Liebowitz (etapa I), os estudantes foram convidados a comparecerem ao Serviço de Psicologia Aplicada (SPA) da UFF Campos, a fim de responderem a Entrevista Clínica Estruturada para os Transtornos do DSM (SCID-5), procedimento que durou cerca de 10 minutos (etapa II). Após passarem pela entrevista clínica, os universitários foram avaliados por meio de uma bateria de testes neuropsicológicos computadorizados, procedimento realizado em um consultório do Serviço de Psicologia Aplicada (SPA) da UFF Campos (etapa III). A avaliação individual durou cerca de 40 minutos.

\subsection{Análise dos dados}

Para a realização das análises estatísticas, utilizou-se o software Statistical Package for the Social Sciences (SPSS para Windows - versão 24.0). Variáveis categóricas foram analisadas através do teste do Qui-quadrado de Pearson. A hipótese de normalidade dos dados foi verificada pelo teste de Kolmogorov-Smirnov. Variáveis numéricas foram analisadas através do teste de Levene para igualdade de variâncias e do teste-t para igualdade de médias, adotando-se um nível de significância $\leq 0,05$. Para o cálculo do tamanho de efeito, utilizou-se o teste $\mathrm{d}$ de Cohen.

\section{RESULTADOS}

Não houve diferença estatisticamente significativa nas variáveis sociodemográficas entre os grupos caso e controle. Observou-se uma prevalência de $83,3 \%$ de indivíduos do sexo feminino $(\mathrm{n}=25)$ e de $16,7 \%$ do sexo masculino $(\mathrm{n}=5)$ na amostra total. No que se refere ao curso de graduação, $70,0 \%$ dos participantes $(n=21)$ eram estudantes de Psicologia, seguido por $16,7 \%(\mathrm{n}=5)$ de História e $6,7 \%(\mathrm{n}=2)$ de Geografia. A amostra também foi composta por 1 estudante de Ciências Sociais e 1 de Economia. Com relação ao uso de medicamentos, constatou-se que apenas 3,3\% $(\mathrm{n}=1)$ da amostra faz uso contínuo de algum psicofármaco. Também foi possível observar a prevalência de comorbidades psiquiátricas em $23,3 \%$ dos participantes $(\mathrm{n}=7)$. Os dados clínicos e sociodemográficos da amostra são apresentados na Tabela 1. 
Tabela 1: Descrição clínica e sociodemográfica da amostra.

\begin{tabular}{|c|c|c|c|}
\hline & $\begin{array}{l}\text { Grupo caso } \\
\quad(n=11)\end{array}$ & $\begin{array}{l}\text { Grupo controle } \\
(n=19)\end{array}$ & $p$ \\
\hline $\begin{array}{l}\text { Sexo } \\
\text { (feminino/masculino) }\end{array}$ & $10 / 1$ & $15 / 4$ & 0,40 \\
\hline Idade (média \pm DP) & $21,90(4,08)$ & $21,95(6,80)$ & 0,31 \\
\hline \multicolumn{4}{|l|}{ Curso } \\
\hline Psicologia & 8 & 13 & \\
\hline Ciências Sociais & - & 1 & \\
\hline História & 2 & 3 & 0,48 \\
\hline Geografia & - & 2 & \\
\hline Economia & 1 & - & \\
\hline \multicolumn{4}{|l|}{$\begin{array}{l}\text { Uso de medicamento } \\
\text { psicotrópico }\end{array}$} \\
\hline Sim & 1 & - & \\
\hline Não & 8 & 16 & 0,39 \\
\hline Já fez & 2 & 3 & \\
\hline \multicolumn{4}{|l|}{ Comorbidade } \\
\hline Nenhuma & 8 & 15 & \\
\hline $\begin{array}{l}\text { Transtorno de Ansiedade } \\
\text { Generalizada }\end{array}$ & 1 & 3 & 0,54 \\
\hline $\begin{array}{l}\text { Transtorno de Ansiedade } \\
\text { e Transtorno Depressivo }\end{array}$ & 1 & 1 & \\
\hline Transtorno Bipolar & 1 & - & \\
\hline
\end{tabular}

Utilizou-se o teste-t de Pearson para a verificação de diferenças entre as médias dos grupos caso e controle. Também foi utilizado o teste $d$ de Cohen para a análise das medidas de tamanho de efeito, as quais fornecem informações sobre a intensidade da relação entre a variável independente e a variável dependente (SHAUGHNESSY, ZECHMEISTER E ZECHMEISTER, 2012). No rastreio da sintomatologia de ansiedade social, observou-se que o grupo caso obteve um escore total médio significativamente maior $(72,91)$ do que o grupo controle $(49,42)$ na LSAS. Com relação à subescala ansiedade social, que inclui as variáveis medo e evitação, identificou-se uma média de 36,18 pontos no grupo caso e de 23,00 pontos no grupo controle $(p=0,020)$. Em ambos os casos da LSAS o tamanho de efeito (d) foi maior que 0,80 , o que é considerado um efeito grande. No que se refere ao desempenho na bateria de testes neuropsicológicos, foi possível observar diferença estatisticamente significativa $(\mathrm{p}=$ $0,009)$ na média do tempo de reação entre os grupos caso $(544,28 \mathrm{~ms})$ e controle $(483,72 \mathrm{~ms})$ através do Go/No-go Task. Também constatamos diferença estatisticamente significativa ( $\mathrm{p}=$ 0,043 ) na média do tempo de reação entre os grupos (caso $=536,11 \mathrm{~ms}$; controle $=469,51$ ms) no teste Oddball. Não foram encontradas diferenças significativas nos demais testes utilizados. As principais médias de desempenho na bateria neuropsicológica são detalhadas na Tabela 2. 
Tabela 2: Desempenho dos grupos na bateria de testes neuropsicológicos.

\begin{tabular}{|c|c|c|c|c|}
\hline & \multicolumn{2}{|c|}{ Média \pm DP } & \multirow[b]{2}{*}{$p$} & \multirow[b]{2}{*}{ d } \\
\hline & $\begin{array}{l}\text { Grupo caso } \\
(n=11)\end{array}$ & $\begin{array}{l}\text { Grupo controle } \\
(n=19)\end{array}$ & & \\
\hline Escore Total na LSAS & $72,91 \pm 21,31$ & $49,42 \pm 31,37$ & $0,036^{*}$ & 0,88 \\
\hline $\begin{array}{l}\text { Escore em ansiedade social } \\
(\text { medo }+ \text { evitação })\end{array}$ & $36,18 \pm 11,64$ & $23,00 \pm 15,24$ & $0,020^{*}$ & 0,97 \\
\hline \multicolumn{5}{|l|}{ Berg's Card Sorting Test } \\
\hline Respostas perseverativas & $37,73 \pm 8,79$ & $36,00 \pm 13,83$ & 0,713 & 0,15 \\
\hline Erros perseverativos & $15,27 \pm 7,17$ & $15,32 \pm 5,74$ & 0,986 & $\begin{array}{c}< \\
0,00\end{array}$ \\
\hline \multicolumn{5}{|l|}{ Go/No-go Task } \\
\hline $\begin{array}{l}\text { Rodada } 1 \text { - Média de precisão } \\
\text { para estímulo } \mathrm{R}\end{array}$ & $0,78 \pm 0,15$ & $0,78 \pm 0,11$ & 0,951 & 0,00 \\
\hline $\begin{array}{l}\text { Rodada } 1-\text { Média do tempo de } \\
\text { reação }(\mathrm{ms}) \text { para estímulo } P\end{array}$ & $544,28 \pm 60,54$ & $483,72 \pm 54,34$ & $0,009 *$ & 1,05 \\
\hline $\begin{array}{l}\text { Rodada } 2 \text { - Média do tempo de } \\
\text { reação (ms) para estímulo } R\end{array}$ & $596,83 \pm 70,41$ & $543,50 \pm 34,77$ & $0,035^{*}$ & 0,96 \\
\hline \multicolumn{5}{|l|}{ Oddball Task } \\
\hline Média do tempo de reação (ms) & $536,11 \pm 94,72$ & $469,51 \pm 75,23$ & $0,043 *$ & 0,78 \\
\hline $\begin{array}{l}\text { Média do tempo de reação }(\mathrm{ms}) \\
\text { para estímulo } 0\end{array}$ & $529,27 \pm 94,53$ & $464,35 \pm 80,58$ & 0,056 & 0,74 \\
\hline $\begin{array}{l}\text { Média do tempo de reação }(\mathrm{ms}) \\
\text { para estímulo } 1\end{array}$ & $\begin{array}{c}605,73 \pm \\
147,82\end{array}$ & $515,89 \pm 118,00$ & 0,078 & 0,67 \\
\hline \multicolumn{5}{|l|}{ Traveling Salesman Problem } \\
\hline $\begin{array}{l}\text { Média de tempo (s) para } 6 \\
\text { pontos }\end{array}$ & $11,87 \pm 4,80$ & $11,76 \pm 5,72$ & 0,959 & 0,02 \\
\hline $\begin{array}{l}\text { Média de tempo (s) para } 20 \\
\text { pontos }\end{array}$ & $22,45 \pm 7,83$ & $20,53 \pm 7,75$ & 0,519 & 0,25 \\
\hline $\begin{array}{l}\text { Média de tempo (s) para } 30 \\
\text { pontos }\end{array}$ & $34,45 \pm 10,87$ & $30,52 \pm 10,45$ & 0,337 & 0,37 \\
\hline
\end{tabular}

Nota: para o tamanho de efeito (d de Cohen), considera-se (CARDOSO, MELO E

FREITAS, 2013):

$\geq 0,20$ : pequeno

$\geq 0,50$ : médio

$\geq 0,80$ : grande

$\geq 1,00$ : muito grande 


\section{DISCUSSÃO}

Alguns dos resultados encontrados neste estudo corroboram os achados da literatura que investigaram a influência dos transtornos de ansiedade sobre o desempenho neuropsicológico em amostras clínicas. Conforme sugerido por Amir et al. (2009), as funções atencionais desempenham um importante papel na manutenção dos sintomas de Transtorno de Ansiedade Generalizada (TAG), reforçando a hipótese de que indivíduos diagnosticados com TAG demonstram um forte viés atencional para estímulos ameaçadores. Em nosso estudo, foram utilizados dois instrumentos para a avaliação da atenção e impulsividade (Go/No-go Task e Oddball Task). A média do tempo de reação em ambos os testes foi maior para os sujeitos do grupo caso, o que sugere possíveis prejuízos em tarefas que demandem atenção seletiva, uma vez que o processamento de informações ao córtex ocorre, em indivíduos saudáveis, de forma acelerada (TEIXEIRA et al., 2015). Nossos resultados também são congruentes com o estudo de Sachs et al. (2004), que demonstraram a ocorrência de déficits no processamento de informação e menor precisão em tarefas de atenção seletiva em indivíduos diagnosticados com TAS.

Em contrapartida, a avaliação de estratégias de resolução de problemas e flexibilidade cognitiva não revelou diferença estatisticamente significativa entre os grupos, como pode ser observado pelas médias de desempenho nas tarefas do Berg's Card Sorting Test (BCST). Nossos resultados contradizem os achados de Cohen et al. (1996), que revelaram prejuízos significativos de indivíduos com ansiedade social em medidas de função executiva, quando comparados a controles saudáveis e pacientes com Transtorno Obsessivo-Compulsivo (TOC). Uma das hipóteses para a divergência de resultados recai sobre a escolha da bateria de testes neuropsicológicos, que embora avaliem funções semelhantes, dispõem de procedimentos técnicos de avaliação diferentes, o que pode influenciar na distinção dos resultados. Nesse sentido, sugere-se que pesquisas futuras examinem as especificidades das funções de planejamento e resolução de problemas em indivíduos ansiosos, de modo a elucidar os potenciais mecanismos deste transtorno sobre o funcionamento executivo.

Em um estudo desenvolvido por Graver e White (2007), os autores compararam o desempenho em tarefas de atenção espacial e resolução de problemas complexos de sujeitos com ansiedade social e controles saudáveis. Observou-se a ocorrência de déficits na resolução de problemas e em habilidades visuoespaciais dos indivíduos com TAS durante a testagem em um contexto de estresse induzido. Apesar de não termos evidenciado diferenças estatisticamente significativas no nosso estudo, verificamos que, em média, o tempo para conclusão das tarefas do Traveling Salesman Problem (TSP) foi maior no grupo caso do que no grupo controle.

Evidencia-se que este estudo trouxe importantes contribuições para a compreensão dos aspectos neuropsicológicos relacionados ao TAS. Contudo, é importante reconhecer que alguns pontos devem ser explorados com maior profundidade em pesquisas futuras. Em primeiro lugar, a nossa capacidade de generalização dos resultados ficou limitada devido ao número de sujeitos. Além disso, uma possível análise comparativa entre os sexos ficou restrita, devido à predominância de mulheres na amostra total, em função do perfil demográfico da universidade onde o estudo foi realizado. Além disso, ressalta-se a necessidade de estudos de adaptação e validação de instrumentos que possibilitem o estabelecimento de uma normativa para a nossa população, o que poderia fornecer parâmetros psicométricos mais confiáveis para a comparação do perfil cognitivo de grupos clínicos e controles saudáveis. 


\section{CONCLUSÃO}

Diante do objetivo de caracterizar o perfil clínico e neuropsicológico de universitários com sintomas de ansiedade social, os resultados deste estudo corroboram os achados da literatura científica que apontam para prejuízos decorrentes do Transtorno de Ansiedade Social em funções neuropsicológicas específicas, como a atenção seletiva, habilidades visuoespaciais e de resolução de problemas complexos. Também observamos diferenças estatisticamente significativas nas médias do tempo de reação entre os grupos caso e controle, o que nos leva a refletir sobre a possível ocorrência de déficits atencionais em indivíduos com critérios diagnósticos para o TAS.

Estudos sobre a eficácia de tratamentos para o Transtorno de Ansiedade Social revelam diferentes abordagens clínicas que incluem o treinamento de habilidades sociais, terapia cognitiva, treinamento de relaxamento, psicoterapia interpessoal, psicoterapia de orientação dinâmica e farmacoterapia (BARLOW, 2016). Entretanto, alguns autores apontam para o fato de que uma parcela substancial de pacientes com transtornos de ansiedade não responde às terapias atualmente disponíveis (FISHER \& DURHAM, 1999; GORMAN, 2003, apud AMIR et al., 2009). Nessa perspectiva, acredita-se que uma das contribuições do nosso estudo foi aprofundar na compreensão dos mecanismos neurocognitivos de indivíduos com ansiedade social, na tentativa de encontrar evidências experimentais que possam ser traduzidas em tratamentos clínicos mais eficazes.

Por fim, faz-se necessário refletir sobre as limitações e prejuízos causados pelo TAS na esfera neurocognitiva, expandindo o olhar do transtorno para além dos aspectos emocionais. Além disso, indaga-se acerca das especificidades clínicas e da alta prevalência de transtornos psiquiátricos na população universitária, a qual está imersa em um contexto de intensa demanda cognitiva. Tais questionamentos devem pautar investigações futuras que sejam capazes de elucidar as nuances neuropsicológicas que se manifestam nos transtornos de ansiedade.

\section{REFERÊNCIAS}

AMIR, N.; BEARD, C.; BURNS, M.; \& BOMYEA, J. Attention modification program in individuals with generalized anxiety disorder. Journal of abnormal psychology, 118(1), 28, 2009. Disponível em: https://www.ncbi.nlm.nih.gov/pmc/articles/PMC2645540/.

ASBAHR, F.R. Transtornos ansiosos na infância e adolescência: aspectos clínicos e neurobiológicos. Jornal de Pediatria, 80(2), 28-34, 2004. Disponível em: https://www.scielo.br/scielo.php?script=sci_arttext\&pid=S0021-75572004000300005.

ASMUNDSON, G.J.; STEIN, M.B.; LARSEN, D.K.; \& WALKER, J.R. Neurocognitive function in panic disorder and social phobia patients. Anxiety, 1(5), 201-207, 1994. Disponível em: https://www.ncbi.nlm.nih.gov/pubmed/9160575.

BAPTISTA, C.A. Estudo da prevalência do transtorno de ansiedade social em estudantes universitários. 2006. 111 p. Dissertação (Mestrado). Ribeirão Preto: Faculdade de Medicina de Ribeirão Preto, Universidade de São Paulo.

BAPTISTA, C.A. et al. Social phobia in Brazilian university students: Prevalence, underrecognition and academic impairment in women. Journal of affective disorders, 136(3), 857-861, 2012. Disponível em: https://linkinghub.elsevier.com/retrieve/pii/S01650327(11)00548-9. 
BARLOW, D.H. Manual clínico dos transtornos psicológicos: Tratamento passo a passo. Porto Alegre: Artmed Editora, 2016.

BEAR, M.F.; CONNORS, B.W.; \& PARADISO, M.A. Neurociências: desvendando o sistema nervoso. Porto Alegre: Artmed Editora, 2008.

BRUCE, S. E. et al. Influence of psychiatric comorbidity on recovery and recurrence in generalized anxiety disorder, social phobia, and panic disorder: A 12-year prospective study. American Journal of Psychiatry, 162, 1179-1187, 2005. Disponível em: https://www.ncbi.nlm.nih.gov/pmc/articles/PMC3272761/.

BURATO, K.R.S.S. (2009). Transtorno de ansiedade social: comportamentos de segurança e evitação. 2009. Dissertação (Mestrado). São Paulo: Universidade de São Paulo.

CARDOSO, T.S.G.; MELLO, C.B.; \& FREITAS, P.M. Uso de Medidas Quantitativas de Eficácia em Reabilitação Neuropsicológica. Psicologia em Pesquisa, 7(1), 121-131, 2013. Disponível em: http://pepsic.bvsalud.org/scielo.php?script=sci_arttext\&pid=S198212472013000100013.

CARLSON, N. R. Fisiologia do comportamento. São Paulo: Manole, 2002.

CASTILLO, A.R.G.; RECONDO, R.; ASBAHR, F.R.; \& MANFRO, G.G. Transtornos de ansiedade. Revista Brasileira de Psiquiatria, 22(Supl. 2), 20-23, 2000. Disponível em: https://www.scielo.br/scielo.php?script=sci_arttext\&pid=S1516-44462000000600006.

COHEN, L.J.; HOLLANDER, E.; DECARIA, C.M.; STEIN, D.J.; SIMEON, D.; LIEBOWITZ, M.R.; \& ARONOWITZ, B.R. Specificity of neuropsychological impairment in obsessive-compulsive disorder: a comparison with social phobic and normal control subjects. The Journal of neuropsychiatry and clinical neurosciences, 1996.

CONSELHO FEDERAL DE PSICOLOGIA. Define e regulamenta o uso, a elaboração e a comercialização de testes psicológicos e revoga a Resolução CFP $n^{0}$ 025/2001. Resolução n. 002 de 2003. Disponível em: http://site.cfp.org.br/wpcontent/uploads/2012/05/resoluxo022003.pdf.

DEL-BEN, C.M.; VILELA, J.A.A.; CRIPPA, J.A.D.S.; HALLAK, J.E.C.; LABATE, C.M.; \& ZUARDI, A.W. Confiabilidade da "Entrevista Clínica Estruturada para o DSM-IV--Versão Clínica" traduzida para o português. Revista Brasileira de Psiquiatria, 2001. Disponível em: https://www.scielo.br/scielo.php?script=sci_arttext\&pid=S1516-44462001000300008.

EATON, W. W.; KRAMER, M.; ANTHONY, J.C.; DRYMAN, A.; SHAPIRO, S.; \& LOCKE, B.Z. The incidence of specific DIS/DSM-III mental disorders: data from the NIMH Epidemiologic Catchment Area Program. Acta Psychiatrica Scandinavica, 79(2), 163-178, 1989. Disponível em: https://onlinelibrary.wiley.com/doi/abs/10.1111/j.16000447.1989.tb08584.x?sid=nlm\%3Apubmed.

GRAVER, C.J., \& WHITE, P.M. Neuropsychological effects of stress on social phobia with and without comorbid depression. Behaviour research and therapy, 45(6), 1193-1206, 2007.

Disponível em: https://www.sciencedirect.com/science/article/abs/pii/S0005796706001720?via\%3Dihub.

HEATON, K.R. et al. Wisconsin Card Sorting Test Manual. Odessa: Psychological Assessment Resources, 1993.

KANDEL, E.; SCHWARTZ, J.; JESSELL, T.; SIEGELBAUM, S.; \& HUDSPETH, A.J. Princípios de Neurociências. Porto Alegre: AMGH Editora, 2014.

KESSLER, R.C. et al. Lifetime and 12-month prevalence of DSM-III-R psychiatric disorders 
in the United States: results from the National Comorbidity Survey. Archives of general psychiatry, $\quad 51(1), \quad 8-19, \quad 1994 . \quad$ Disponível https://jamanetwork.com/journals/jamapsychiatry/article-abstract/496456.

MAGEE, W. J.; EATON, W.W.; WITTCHEN, H.U.; MCGONAGLE, K. A.; \& KESSLER, R.C. Agoraphobia, simple phobia, and social phobia in the National Comorbidity Survey. Archives of General Psychiatry, 53, 159-168, 1996. Disponível em: https://jamanetwork.com/journals/jamapsychiatry/article-abstract/497537.

LIMA, M.S.D. Epidemiologia e impacto social. Revista Brasileira de psiquiatria, 21, 01-05, 1999. Disponível em: https://www.scielo.br/scielo.php?script=sci_arttext\&pid=S151644461999000500002.

MUELLER, S.T. PEBL: The Psychology experiment building language (Version 0.14) [Computer experiment programming language]. 2014. Disponível em: http://pebl.sourceforge.net.

PIES, R.W. Clinical manual of psychiatric diagnoses and treatment: a biopsychosocial approach. Washington: American Psychiatric Press, 1994.

SACHS, G.; ANDERER, P.; MARGREITER, N.; SEMLITSCH, H.; SALETU, B.; \& KATSCHNIG, H. P300 event-related potentials and cognitive function in social phobia. Psychiatry Research: Neuroimaging, 131(3), 249-261, 2004. Disponível em: https://www.sciencedirect.com/science/article/abs/pii/S0925492704000575.

SANTOS, L.F.; LOUREIRO, S.R.; CRIPPA, J.A.; \& OSÓRIO, F.L. Can the Liebowitz Social Anxiety Scale-self-report version be used to differentiate clinical and non-clinical SAD groups among Brazilians? PLOS ONE, 10(3), e0121437, 2015. Disponível em: https://www.ncbi.nlm.nih.gov/pmc/articles/PMC4374964/.

SCHNEIER, F.R.; JOHNSON, J.; HORNIG, C.D.; LIEBOWITZ, M. R.; \& WEISSMAN, M.M. Social phobia: Comorbidity and morbidity in an epidemiologic sample. Archives of General Psychiatry, 49, 282-288, 1992. Disponível em: https://jamanetwork.com/journals/jamapsychiatry/article-abstract/495695.

SHAUGHNESSY, J.J.; ZECHMEISTER, E.B.; \& ZECHMEISTER, J.S. Metodologia de Pesquisa em Psicologia. Porto Alegre: AMGH Editora, 2012.

SUTTERBY, S. Neuropsychological Functioning In Social Phobia [dissertation]. Orlando: University of Central Florida, 2009.

TALÃO, F.C.D.L.M.; DE SOUZA, J.C.; ALVES, M.C.C.; MACHADO, F.D.A.L.; \& SHIMOYA, A. Fatores que podem contribuir para transtornos psiquiátricos nos habitantes de Cardoso Moreira-RJ. Biológicas \& Saúde, v. 10, n. 32, p. 36-50, 21 fev. 2020. Disponível em: https://ojs3.perspectivasonline.com.br/biologicas_e_saude/article/view/1908.

TEIXEIRA, D.; CALOMENI, M.R.; AZEVEDO, L.; ALMEIDA, M.; \& ARÊAS NETO, N.T. O slackline como atividade promotora da atenção concentrada em escolares da rede básica de educação em Campos dos Goytacazes - RJ, moradores da comunidade Tamarindo. Biológicas \& Saúde, v. 5, n. 18, 24 nov. 2015. Disponível em: https://ojs3.perspectivasonline.com.br/biologicas_e_saude/article/view/798.

WHITBOURNE, S.K.; \& HALGIN, R.P. Psicopatologia. Porto Alegre: AMGH Editora, 2015.

WHITE, P. Biopsychosocial medicine: an integrated approach to understanding illness. New York: Oxford University Press, 2005. 